\title{
Joint Decoding of Multiple-Description Network-Coded Data
}

\author{
Lana Iwaza*, Michel Kieffer*, Leo Liberti ${ }^{\ddagger}$, and Khaldoun Al-Agha ${ }^{\S}$ \\ ${ }^{*}$ L2S - CNRS - SUPELEC - Univ Paris-Sud, 3 rue Joliot-Curie, F-91192 Gif-sur-Yvette Cedex \\ Email: lana.iwaza@1ss.supelec.fr

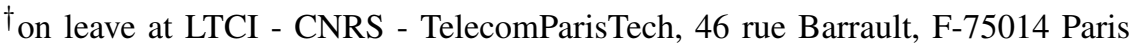 \\ Email: kieffer@1ss.supelec.fr \\ ${ }_{\ddagger}^{\ddagger}$ LIX - Ecole Polytechnique, F-91128 Palaiseau \\ Email: leo.liberti@lix.polytechnique.fr \\ §LRI - CNRS - Univ Paris-Sud, Bâtiment 490 - Université Paris-Sud, F-91405 Orsay cedex \\ Email: alagha@lri.fr
}

\begin{abstract}
This paper considers a transmission scheme combining multiple description coding (MDC) and network coding (NC). Our aim is to benefit from the property of MDC to provide progressive quality improvement with the number of received packets and from the efficient use of network resources provided by NC.

MDC is performed either via frame expansion (NC-MDC-F) or via correlating transform (NC-MDC-T). With NC-MDC-F, when not enough $\mathrm{NC}$ have been received, reconstruction may be done via mixed integer quadratic programming (MIQP). With NCMDC-T, the reconstruction involves simple Gaussian elimination.

In both cases, a good robustness to missing NC packets is observed. When the number of missing packets is small, NCMDC-F provides better SNR thanks to a reduction of a part of the quantization noise. The price to be paid is a decreased robustness to missing packets. When the number of lost packets increases, a reconstruction is still possible with NC-MDC-F for some packets, even if the number of missing packets is larger than $n-k$, the number of introduced redundancy packets.

A typical application context is multicasting of multimedia contents with delay constraints. Users with very good channel conditions will benefit from the reception of redundant packets with NC-MDC-F, whereas these packets are useless with NCMDC-T or with error-correcting NC. ${ }^{1}$
\end{abstract}

Index Terms-Consistent reconstruction; Frame expansions; Multiple description coding; Network coding; Quantization.

\section{INTRODUCTION}

Consider a mixed wired and wireless network in which some compressed multimedia stream has to be transmitted from a server to a set of receivers. The recovery of the transmitted data may be significantly affected by the timevarying characteristics (capacity, delays, or losses) of each communication link, especially in the case of constraints on the delivery delay. Thus, the quality of the decoded stream at each receiver is far from being deterministic.

Layered or scalable coding techniques [1] have been proposed to ensure a minimum Quality of Service (QoS) to users with poor channel conditions and better QoS to users with

\footnotetext{
${ }^{1}$ This work was partly supported by the Digiteo PASO, NC2!!, and DIMLSC SWAN projects.
}

better conditions. In such coding schemes, compression is performed to allow encoded data packets to be partitioned into several priority layers. At receiver side, the quality of decoded data increases with the number of received layers. This type of coding mechanism is very efficient when combined with networks allowing differentiated service protocols, such as DiffServ [2] or combined with a priority encoding transmission system [3]. The main drawback of the layered approach comes from the fact that a low-priority layer is useless unless all its associated higher-priority layers have been received. For networks with links prone to losses and delay-sensitive applications for which retransmission is difficult, this may lead to a waste of the available resources. Unequal error protection (UEP) can be seen as a way to maintain an acceptable quality in the face of losses, as described in [4].

In presence of packet losses, erasure-correcting codes at intermediate protocol layers have been proposed, see, e.g., [5]. The approach is efficient as soon as enough packets have been received, but is useless when the erasure-correcting capacity is overflowed. Multiple Description Coding (MDC) techniques, see [6] and the references therein, represent an alternative approach to cope with lossy networks. Structured redundancy is introduced during compression to generate packets which may be partitioned in equally important descriptions of the source data. Reconstruction with a given quality is possible with the reception of one description and gradually improves with the number of received descriptions. Multiple description may be performed using adapted quantization [7], correlating transforms [8], frame expansions [9], etc. Optimized routing techniques have been proposed in [10] with the concept of Rainbow Networks, where descriptions are assimilated as colors, the goal being to determine which combination of colors has to be associated to each link of the network to maximize reconstruction quality at the various receivers. Nevertheless, the proposed optimization approach is centralized and requires some knowledge of the network topology.

Network coding (NC), introduced in [11], has been proposed to better use the network resources. With this approach, 
intermediate nodes of the network are allowed to combine incoming packets instead of simply forwarding them. The throughput in multicast networks may then be increased to reach the max-flow capacity between the source and each destination node [12]. Practical aspects of NC have been considered in [13], where deterministic linear combination of incoming packets are performed. In [14], random linear network coding (RLNC) has been introduced as a way of allowing decentralized coding operations as well as robustness against network changes and link failures.

By construction, RLNC is quite robust against losses of coded packets. A receiver has only to wait until enough informative packets have been received to perform decoding. Nevertheless, in many situations, the NC network may time out [15], leaving receivers without enough packets to perform network decoding. This issue has been addressed with a networking perspective in [16], where a loss-tolerant protocol for broadcasting using NC (DRAGONCAST) has been proposed. This technique allows a real-time decoding of the network-coded packets, and an adjustment of NC transmission rate. A channel coding perspective is considered in [13], where error-control codes in network-coded systems have been proposed and the usual link-by-link error-correction approach is used. In [17], [18], a rank-metric RLNC approach is introduced to address the problems of errors or missing network-coded packets. This approach provides a maximum distance separable network code, where missing packets are recovered using a process similar to Reed-Solomon decoding. However, when the number of errors and erasures exceeds the correction capacity, no reconstruction can be achieved.

Several alternative joint source-network coding approaches have been proposed recently, trying to get the best of layered or MDC techniques and NC. A first attempt to combine NC with rainbow network flow is presented in [19] where higher network throughput is achieved compared to the original rainbow network flow solution, however, only intra-layer NC is performed. In [20] and [21] techniques to perform intra- and inter-layer NC are proposed. Concatenated MDC and RLNC have been introduced in [22], where a concatenation of an inner network code and an outer PET code is considered to allow receivers with low resources to network decode the most important packets without requiring all packets to be received. In [23] UEP is combined with NC. Source packets are again grouped into priority classes. NC is performed in such a way that a small amount of network-coded packets allow to decode the highest priority class. In [24], the correlation existing between packets transmitted, e.g., by neighboring sensors of a sensor network is exploited to perform an approximate decoding when not enough network-coded packets have been received to perfectly perform network decoding. The influence of the size of the Galois field in which quantization and NC are performed, is studied and an optimal size in terms of reconstruction noise is evaluated.

This paper considers NC of packets which have been encoded using MDC schemes. Our aim is to show that redundancy introduced before quantization using MDC via frame expansion [25] may be efficiently used at receiver side to decode network-coded packets and to mitigate part of the quantization noise. Focusing on the case of missing network-coded packets, this paper shows that the estimation of the original packets using the received combinations and taking into account the redundancy introduced during frame expansion can be obtain via the solution of a mixed integer quadratic program. This provides some robustness to packet losses, with a quality of the reconstructed packets increasing with the number of received packets. When considering MDC via correlating transform followed by $\mathrm{NC}$, the reconstruction in presence of missing combinations may be done as in [24]. However, contrary to MDC via frame expansion, as soon as enough packets are received to be able to perform decoding, there is no advantage in receiving more packets.

The proposed coding scheme is presented in Section II. The way MDC data is recovered after NC is illustrated in Section III. Section IV compares NC of MDC packets via frame expansion (NC-MDC-F) and NC of MDC packets via correlating transform (NC-MDC-T), with a reconstruction method from [24]. Simulation results are described in Section V before drawing some conclusions in Section VI.

\section{CODING SCHEME}

Consider some overlay network with a source, several intermediate node, and receiver nodes. Figure 1 represents the part of the communication chain (including the network) between the source and a given receiver.

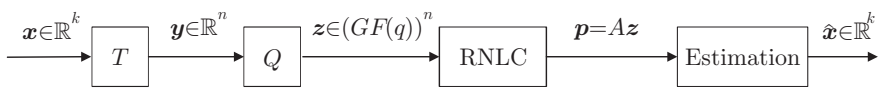

Fig. 1. Block diagram of the proposed system

A source vector $x \in \mathbb{R}^{k}$ is expanded using a frame expansion matrix $T$, producing the vector $y \in \mathbb{R}^{n}$, with $n>k$. Each entry of $y$ is quantized with a $q$-level uniform scalar quantizer to get a vector of indexes $z \in(G F(q))^{n}$. Each index is then put in a separate packet and the $n$ packets are transmitted over the network. RLNC is performed at intermediate nodes of the network. At receiver side, a set of $m$ network-coded packets is obtained. An estimate $\widehat{x}$ of $x$ from the received packets grouped in a vector $p$ using the various constraints imposed by the system is finally evaluated. In what follows, each step in Figure 1 is described to evidence the constraints linking the variables of the system, which will help obtaining $\widehat{x}$ at receiver nodes.

\section{A. Multiple description via frame expansion}

MDC is performed via a real-valued frame expansion. A real-valued frame of $\mathbb{R}^{k}$ [26] is a set of $n>k$ vectors $\left\{\varphi_{i}\right\}_{i=1 \ldots n}$ such that there exists $A>0$ and $B<\infty$ (the frame bounds) satisfying for all $x \in \mathbb{R}^{k}$,

$$
A\|x\|^{2} \leq \sum_{i=1}^{n}\left\langle x, \varphi_{i}\right\rangle^{2} \leq B\|x\|^{2},
$$


where $\langle\cdot, \cdot\rangle$ is the inner product of $\mathbb{R}^{k}$. When $A=B$, the frame is tight. The frame operator $T$ associated to $\left\{\varphi_{i}\right\}_{i=1 \ldots n}$ is the linear operator from $\mathbb{R}^{k}$ to $\mathbb{R}^{n}$ defined as

$$
(T x)_{i}=\left\langle x, \varphi_{i}\right\rangle, i=1 \ldots n .
$$

For any $x \in \mathbb{R}^{k}$, the frame operator $T$ produces a vector

$$
y=T x \in \mathbb{R}^{n} .
$$

The redundancy rate introduced by a tight frame expansion is $r=n / k$.

\section{B. Quantization}

A $q$-level uniform scalar quantization $Q$ with step size $\Delta$ is performed on each entry $y$, resulting in a vector of quantization indexes $z$, whose entries $z_{i}=Q\left(z_{i}\right) \in G F(q)$. The quantization intervals are $[(i-q / 2) \Delta,(i-q / 2+1) \Delta]$, $i=0 \ldots q-1$. The reconstruction levels are chosen at the middle of the quantization intervals, $r_{i}=(i-q / 2+1 / 2) \Delta$, $i=0 \ldots q-1 . \Delta$ is chosen large enough to ensure that all entries of $y$ fall inside a quantization interval. The inverse quantizer $Q^{-1}$ takes a quantization index $z_{i}$ and associates a reconstruction

$$
Q^{-1}\left(z_{i}\right)=\alpha z_{i}+\beta
$$

with $\alpha=\Delta$ and $\beta=(-q+1) \Delta / 2$. Since all reconstruction levels have been taken at the middle of the quantization intervals, $Q^{-1}\left(z_{i}\right)$ satisfies

$$
\begin{aligned}
y_{i}-Q^{-1}\left(z_{i}\right) & \leq \Delta / 2, \\
-y_{i}+Q^{-1}\left(z_{i}\right) & \leq \Delta / 2 .
\end{aligned}
$$

\section{Network Coding}

The vector of quantized indexes $z$ is then transmitted over the network. Each entry of $z$ is transmitted in a separate packet. RLNC is performed at the intermediate nodes of the network. Assume that the $\ell$-th receiver has access to $m$ independant packets $p_{\mu} \in G F(q), \mu=1 \ldots m$, with $m \leq n$. Since these packets have been network coded, the relation between $p=$ $\left(p_{1}, \ldots, p_{m}\right)^{T}$ and $z$ may be written as follows

$$
p=A z
$$

where the network matrix $A \in(G F(q))^{m \times n}$ is the matrix of global NC coefficients. The coefficients of $A$ may be recovered from the headers of each received packet [27]. Usually, $m=n$ packets have to be received in order to recover the uncoded packets. However, even if $m=n, A$ is not necessarily of full rank $n$. Moreover, when not enough packets have been received, $A$ is not of full rank $n$ and the uncoded packets cannot be recovered directly.

\section{ESTIMATION OF THE SOURCE VECTOR}

An estimate $\widehat{x}$ of the source vector $x$ based on the received network-coded packets $p$ and using the fact that $x$ has been expanded into $y$ before quantization and transmission has to satisfy a system of equations and inequalities derived from (3), (5), (6), and (7). Gathering all constraints, one gets

$$
\begin{cases}y_{i}=\sum_{j=1}^{k} t_{i, j} x_{j}, & i=1 \ldots n \\ y_{i}-\left(\alpha z_{i}+\beta\right) \leq \Delta / 2, & i=1 \ldots n \\ -y_{i}+\left(\alpha z_{i}+\beta\right) \leq \Delta / 2, & i=1 \ldots n \\ z_{i} \in\{0, \ldots, q-1\} & i=1 \ldots n \\ p_{\mu}=\sum_{j=1}^{n} a_{\mu j} z_{j}, & \mu=1 \ldots m\end{cases}
$$

where $T=\left(t_{i j}\right)_{i=1 \ldots n, j=1 \ldots k}$ and $A=\left(a_{\mu j}\right)_{\mu=1 \ldots m, j=1 \ldots n}$ have been defined in Section II. In the last line of (8), all operations are done in $G F(q)$. This system contains $n+m$ equations, $2 n$ inequalities, and $2 n+k$ unknows, namely $x \in$ $\mathbb{R}^{k}, y \in \mathbb{R}^{n}$, and $z \in(G F(q))^{n}$. Due to the quantization, $x$ cannot be recovered exactly, even if $A$ is full rank.

\section{A. Enough network-coded packets have been received}

When $A$ is full rank $n$ (enough network coded packets have been received), $A$ may be inverted to get an estimate $\widehat{y}$ for $y$

$$
\widehat{y}=\alpha A^{-1} p+\beta \text {. }
$$

Then, a least-squares estimate $\widehat{x}$ for $x$ is easily obtained as

$$
\widehat{x}=\left(T^{T} T\right)^{-1} T^{T} \widehat{y} .
$$

\section{B. Network-coded packets are missing}

When $A$ is not of full rank $n, A$ cannot be inverted. Since there is not a unique $\widehat{x}$ satisfying (8), one may search for an estimate of minimum norm

$$
\widehat{x}=\arg \min _{x \text { satisfying }(8)} x^{T} x
$$

Nevertheless, this optimization problem is quite hard to solve, since it involves continuous-valued variables and variables belonging to a Galois field. Moreover, equality and inequality constraints have to be considered. Assume that the Galois field is of prime size ( $q$ is prime, extension fields are considered in the appendix), one may transform the last line in (8) by introducing $m$ slack integer variables $s_{\mu}$ as follows

$$
p_{\mu}=\sum_{j=1}^{n} a_{\mu j} z_{j}+q s_{\mu}, \mu=1 \ldots m
$$

to get $m$ equations involving standard integer additions and multiplications.

Solving (11) with the modified system where (12) is put in (8) consists in solving a mixed integer quadratic problem (MIQP). This kind of minimization problem may be modeled using AMPL [28]. Since this MIQP only involves convex quadratic forms, it can be solved using CPLEX [29]. CPLEX implements a Branch-and-Bound (BB) search whose node bounds are computed by solving a continuous relaxation of the MIQP. Since this relaxation is convex, it can be solved using either a modified simplex method or a barrier method (in case the quadratic terms are in the constraints). The whole BB solution algorithm runs in exponential time in the worst case. 


\section{Alternative MDC SCheme}

In [24], an approximate decoding scheme based on existing correlation between quantized packets is proposed. Several correlated sources are considered and no frame expansion is performed on the source signals before quantization. The correlation between source symbols is still present on the quantized indexes $z$ and translates into the fact that there exists some known matrix $D$ of size $(n-k) \times n$ such that

$$
D z=0 \text {. }
$$

$D$ plays a role similar to a parity-check matrix of a traditional error-correcting code. The relation (13) may then be exploited at receivers which do not have access to enough network-coded packets. Consider a network matrix $A$ of dimension $m \times n$, with $m \leq n$. If there exists a submatrix $B^{\prime}$ of size $n \times n$ of

$$
B=\left(\begin{array}{c}
A \\
D
\end{array}\right)
$$

that is of full rank $n$, then $B^{\prime}$ may be inverted to get an estimate $\widehat{z}$ of the quantized source samples.

The probability that such matrix $B^{\prime}$ exists may be shown to be as follows.

$$
\begin{aligned}
& \operatorname{Pr}\left(\operatorname{rank}\left(\begin{array}{c}
A \\
D
\end{array}\right)=n\right)= \\
& \left\{\begin{array}{c}
0 \text { if } m<k, \\
\prod_{i=1}^{k}\left(1-q^{-i}\right) \text { if } m=k, \\
\sum_{\substack{i_{1} \ldots i_{m-k}=1 \\
i_{1}<i_{2}<\cdots<i_{m-k}}}^{m} q^{-k+i_{1}-1} \ldots q^{-k+i_{m-k}-(m-k)} \\
\prod_{i=1}^{k}\left(1-q^{-i}\right) \text { if } m>k .
\end{array}\right.
\end{aligned}
$$

To compare the proposed approach with that in [24], quantization of the source vector $x \in \mathbb{R}^{k}$ is performed first to get quantization indexes $y^{\prime} \in G F(q)^{k}$. Then a full-rank $k$ correlating transform $T_{c} \in G F(q)^{n \times k}$ is used to get a vector $z^{\prime} \in G F(q)^{n}$. The matrix $D$ is chosen to be orthogonal to $T_{c}$. NC is then performed and modeled as in Section II-C to get packets

$$
p^{\prime}=A z^{\prime} .
$$

Estimation of $z^{\prime}$ is performed via classical Gaussian elimination as long as there exists some invertible submatrix $B^{\prime}$ of $B$ in (14). If no invertible $B^{\prime}$ can be found, no reconstruction can be obtained.

Compared to the approach introduced in Section II, here, redundancy is introduced after quantization. The decoding process is then quite simple, since it involves only Gaussian elimination.

\section{SimUlation RESUlTS}

In what follows, $k=4$ and $n=7$. The source generates vectors of $k$ independent and identically distributed Gaussian samples with zero-mean and variance $\sigma^{2}=1$, cropped to $\pm 3 \sigma$.

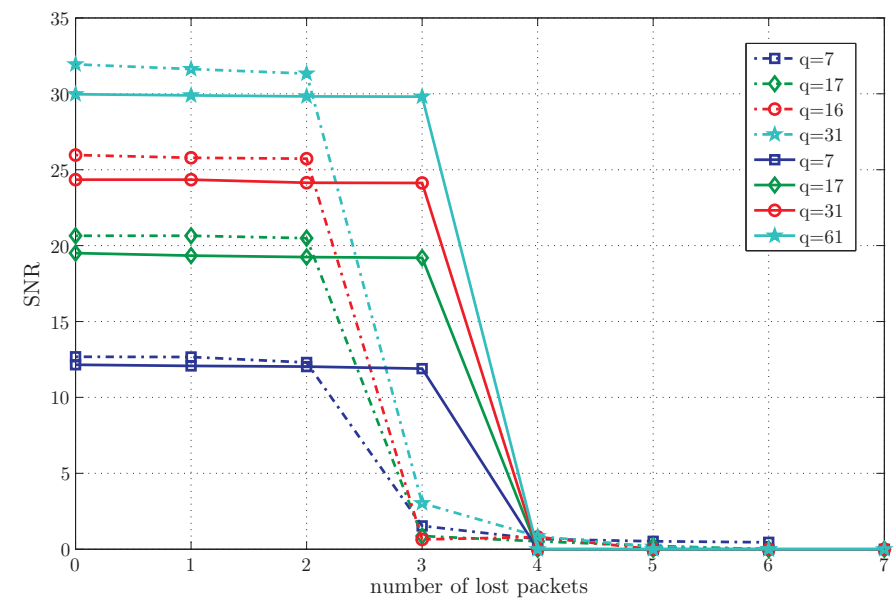

Fig. 2. SNR (in $\mathrm{dB}$ ) as a function of the number of lost packets and the size of the considered Galois field, NC-MDC-F (dashed) and NC-MDC-T (plain)

Then $T$ is built with Lines 2 to 5 of an $n \times n$ DCT transformation matrix. The uniform quantizer with quantization cells partitioning the interval $[-3 \sigma, 3 \sigma]$ is chosen $^{2}$. Quantization with $q \in\{7,17,31,61\}$ quantization intervals is considered, leading to quantization indexes in $G F(q)$. The $m \times n$ network matrix $A$ is chosen at random with $m \leq n$ to simulate the effect of network coding.

The transmission scheme described in Section IV is also simulated with the same source, $k$, and $n$. The corresponding reconstruction scheme is denoted as NC-MDC-T (for Network Coding with MDC via correlating Transform). Like NC-MDCF, quantization with $q \in\{7,17,31,61\}$ quantization intervals is considered, leading to quantization indexes in $G F(q)$. A fixed $n \times k$ random matrix $T_{c} \in G F(q)$ with full rank $k$ is chosen to introduce redundancy. An $(n-k) \times n$ matrix $D$ with full rank $n-k$ is deduced from $T_{c}$. In both cases, simulations results are averaged over 1000 realizations of the source and of the network matrix $A$.

Figure 2 represents the average Signal-to-Noise Ratio (SNR)

$$
S N R_{\mathrm{dB}}=10 \log _{10} \frac{\|x\|^{2}}{\|x-\widehat{x}\|^{2}}
$$

resulting from the reconstruction of the transmitted message as a function of the number of lost packets for various sizes of the Galois field used in quantization and $\mathrm{NC}$ operations. NC-MDC-F is less robust to erasures than NC-MDC-T, since one more erasure is tolerated. However, when enough packets have been received, the reconstruction quality is better using NC-MDC-F. In fact, the frame expansion allows to reduce the effect of quantization noise. This effect is not obtained with the correlating transform. Figures 3 and 4 provide the evolution of the decoding errors (when $\widehat{z} \neq z$ ) as a function of the number of lost packets for both schemes.

With the NC-MDC-T scheme, when the number of losses is larger than the number of redundant packets, i.e., larger

\footnotetext{
${ }^{2}$ Depending on the rate, better rate-distortion performance may be obtained by adjusting more carefully the boundaries of the quantization domain
} 


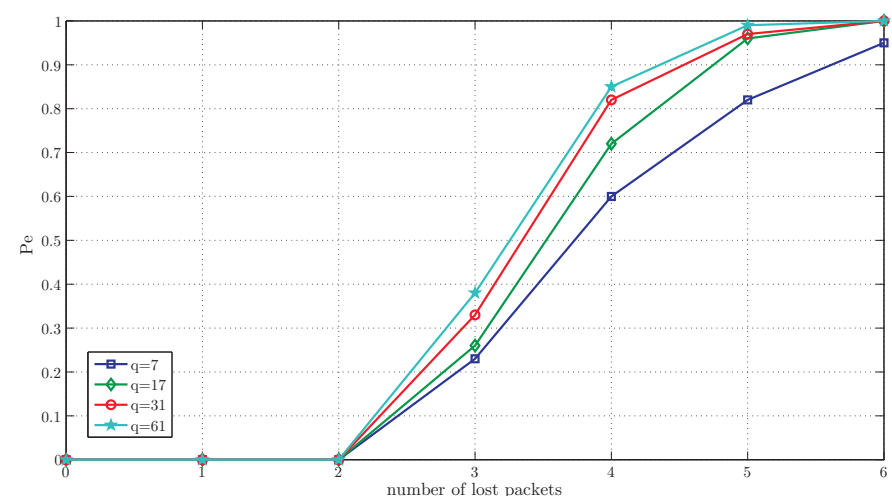

Fig. 3. NC-MDC-F: Proportion of erroneously reconstructed packets as a function of the number of lost packets and of the size of the Galois field

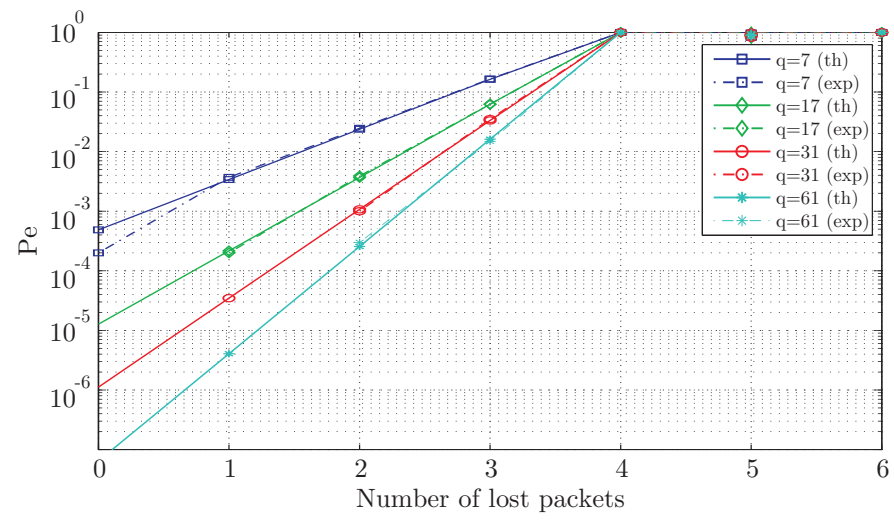

Fig. 4. NC-MDC-T: Proportion of erroneously reconstructed packets as a function of the number of lost packets and the size of the Galois field (th: evaluated using (15), exp: experimental)

than $n-k=3$ in our case, the decoding error probability is one. The reconstruction therefore is impossible. Whereas in the case of NC-MDC-F, a non-zero fraction of the transmitted packets can still be correctly decoded, even if the number of missing packets is larger than the one being introduced during the frame expansion process. The decoding error probability increases smoothly. When 3 packets have not been received, about $70 \%$ of the packets are still correctly decoded with NCMDC-F and the poor SNR observed in Figure 2 is mainly due to erroneously recovered source samples. Being able to detect when samples were not reconstructed correctly may significantly improve the performance when many NC packets are lost.

Figure 5 shows improvements provided by the MIQP reconstruction, by comparing a NC-MDC-F scheme and a scheme where MDC-F is performed and $\mathrm{NC}$ is done on packets corresponding to the same description. In the latter case, it is assumed that when a packet is lost, the whole description is lost. No MIQP reconstruction is possible. One sees that MIQP allows to get almost the maximal reconstruction quality even if some packets are lost. However, when too much packets are lost, the MDC-F scheme provides much smoother SNR decrease than the NC-MDC-F.

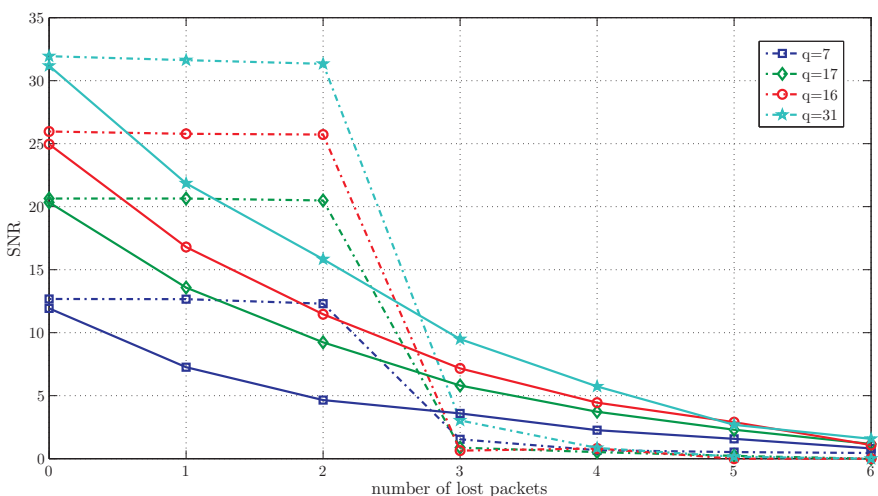

Fig. 5. NC-MDC-F (dashed) compared to a MDC-F scheme (plain) where $\mathrm{NC}$ is only performed between packets of the same description

\section{CONCLUSION}

The paper introduces a transmission scheme combining MDC and NC. Multiple descriptions have been obtained either using a frame expansion (NC-MDC-F) or using a correlating transform (NC-MDC-T). In the first case, the reconstruction is performed via MIQP. In the second case, a reconstruction algorithm derived from [24] has been employed. In both cases, a good robustness to missing $\mathrm{NC}$ packets has been observed. When the number of lost packets is small, the NC-MDC$\mathrm{T}$ provides better SNRs thanks to a partial reduction of the quantization noise. The price to be paid is a decreased robustness to losses. When the number of lost packets increases, a reconstruction is still possible for some packets, even if the number of losses is larger than $n-k$, the number of redundant packets.

When combining packets containing only samples from the same description, least-squares reconstruction techniques may be employed when some descriptions are missing. This provides smooth performance degradation when the number of lost descriptions increases. This property is somewhat lost with NC-MDC-F. An optimization of the way NC is performed has to be found to get smoother performance degradation.

\section{APPENDIX}

\section{A. Reconstruction in $G F\left(q^{r}\right)$}

Since the received packets have been network-coded, the relation between $p=\left(p_{1}, \ldots, p_{m}\right)$ and the quantized indexes $z=\left(z_{1}, \ldots, z_{n}\right)$ can be expressed as

$$
p=A z
$$

or equivalently

$$
p_{j}=\sum_{k=1}^{n} a_{j, k} z_{k}, 1 \leq j \leq m,
$$

where all variables and operations are in $G F\left(q^{r}\right)$.

Equation 17 holds in $G F\left(q^{r}\right)$. To express it in $\mathbb{Z}$, one uses the fact that elements of $G F\left(q^{r}\right)$ may be viewed as polynomials of $G F(q)[D]$. Let $A(D)$ be the network coding matrix of polynomials, each coefficient $a_{j, k} \in G F\left(q^{r}\right)$ of 
$A$ in (17) being represented by a polynomial $a_{j, k}(D)$. Since $G F\left(q^{r}\right)$ is isomorphic to $G F(q)[D] / g(D) G F(q)[D]$, where $g(D)$ is a generator polynomial of $G F\left(q^{r}\right)$, (17) can be expressed in $G F(q)[D]$ as

$$
p(D)=A(D) z(D)+s(D) g(D),
$$

where $p(D)=\left(p_{1}(D), \ldots, p_{m}(D)\right)^{T}, z(D)=$ $\left(z_{1}(D), \ldots, z_{n}(D)\right)^{T}$, and $s(D)=\left(s_{1}(D), \ldots, s_{m}(D)\right)^{T}$ is a vector of slack polynomials. All operations in (19) are now in $G F(q)$.

To express (19) in $\mathbb{Z}[D]$, an additional vector of slack polynomials $\lambda(D)=\left(\lambda_{1}(D), \ldots, \lambda_{m}(D)\right)^{T}$ needs to be introduced. Then, (19) can be expressed as

$$
p(D)=A(D) z(D)+s(D) g(D)+\lambda(D)
$$

where all operations are in $\mathbb{Z}$ and with $\lambda_{j, i} \in \mathbb{Z}, 0 \leq s_{j, i} \leq$ $q-1,0 \leq z_{k, i} \leq q-1,0 \leq a_{j, k, i} \leq q-1,0 \leq g_{i} \leq$ $q-1,0 \leq p_{j, i} \leq q-1$. The subscript $i$, for example in $s_{j, i}$, indicates the coefficient of degree $i$ of the polynomial $s_{j}(D)$. Consequently, (20) can be expressed as

$$
p_{j}(D)=\sum_{k=1}^{n} a_{j, k}(D) z_{k}(D)+s_{j}(D) g(D)+\sum \lambda_{j, i} D^{i}
$$

with $1 \leq j \leq m$. Consider the term of degree $\ell$ in (21)

$$
p_{j, \ell}=\sum_{k=1}^{n} \sum_{i=0}^{\ell} a_{j, k, i} z_{k, \ell-i}+\sum_{i=0}^{\ell} s_{j, i} g_{\ell-i}+\lambda_{j, \ell}
$$

In particular, when $\ell>\operatorname{deg}\left(p_{j}(D)\right)$, one gets

$$
0=\sum_{k=1}^{n} \sum_{i=0}^{\ell} a_{j, k, i} z_{k, \ell-i}+\sum_{i=0}^{\ell} s_{j, i} g_{\ell-i}+\lambda_{j, \ell} .
$$

An estimate $\widehat{x}$ of the source vector $x$ based on the received network-coded packets $p$ and using the fact that $x$ has been expanded into $z$ before quantization and transmission may be obtained by minimizing $x^{T} x$ under the following set of constraints

$$
\begin{cases}y_{u}=\sum_{v=1}^{k} t_{u, v} x_{v}, & u=1 \ldots n \\ y_{u}-\left(\alpha z_{u}+\beta\right) \leq \Delta / 2, & u=1 \ldots n \\ -y_{u}+\left(\alpha z_{u}+\beta\right) \leq \Delta / 2, & u=1 \ldots n \\ p_{j, \ell}=\sum_{k=1}^{n} \sum_{i=0}^{\ell} a_{j, k, i} z_{k, \ell-i}+\sum_{i=0}^{\ell} s_{j, i} g_{\ell-i}+\lambda_{j, \ell}, & \\ \quad j=1 \ldots m, \ell=1, \ldots, 2(r-1) & \\ 0 \leq z_{u} \leq q-1 & u=1 \ldots n \\ 0 \leq s_{j, i} \leq q-1 & \\ (q-1)[1-\ell(q-1)(n+1)] \leq \lambda_{j, \ell} \leq 0 & \end{cases}
$$

In fact $\operatorname{deg}\left(s_{j}(D) G(D)\right)=\operatorname{deg}\left(A(D) z_{j}(D)\right)=2(r-1)$. Since $\operatorname{deg}(g(D))=r$, then $\operatorname{deg}\left(s_{j}(D)\right) \leq r-2$. In the worst case, $(r-1) m$ slack variables $s_{j, i}$ need to be introduced, in addition to $(2 r-1) m$ slack variables $\lambda_{j, \ell}$. The total number of slack variables to introduce is then equal to $(r-1) m+$ $(2 r-1) m=(3 r-2) m$, whereas in the particular case of $r=1$ only $m$ slack variables needed to be introduced.

\section{REFERENCES}

[1] M. Ghanbari, "Two-layer coding of video signals for VBR networks," IEEE J Sel Areas Commun, vol. 7, no. 5, pp. 771-781, 1989.

[2] S. Blake, D. Black, M. Carlson, E. Davies, and Z. Wang, "An architecture for differentiated services," RFC-2475, Tech. Rep., 1998.

[3] A. Albanese, J. Blomer, J. Edmonds, M. Luby, and M. Sudan, "Priority encoding transmission," IEEE Trans. Inf. Th., vol. 42, no. 6, pp. 17371744, 1996.

[4] U. Horn, K. Stuhlmuller, M. Link, and B. Girod, "Robust internet video transmission based on scalable coding and unequal error protection," Sig. Proc.: Im. Comm., vol. 15, pp. 77-94, 1999.

[5] J. Paavola, H. Himmanen, T. Jokela, J. Poikonen, and V. Ipatov, "The performance analysis of MPE-FEC decoding methods at the DVB-H link layer for efficient IP packet retrieval," IEEE Trans. Broadcasting, vol. 53, no. 1, pp. 263-275, 2007.

[6] V. K. Goyal, "Multiple description coding: Compression meets the network," IEEE Sig. Proc. Mag., vol. 18, pp. 74-93, 2001.

[7] O. A. Lotfallah and S. Panchanathan, "Adaptive multiple description coding for internet video," IEEE Trans. Acoust., Speech, Sig. Proc., vol. 5, pp. 732-735, 2003.

[8] V. K. Goyal, "Beyond traditional transform coding," Ph.D. dissertation, University California, Berkeley, 1998.

[9] V. K. Goyal, J. Kovacevic, and M. Vetterli, "Multiple description transform coding: Robustness to erasures using tight frame expansions," August 1998, p. 408

[10] N. Sarshar and X. Wu, "Joint network-source coding: An achievable region with diversity routing," in CoRR abs/cs/0511048, 2005.

[11] R. Ahlswede, N. Cai, S.-Y. R. Li, and R. W. Yeung, "Network information flow," IEEE Trans. Inf. Th., vol. 46, pp. 1204-1216, 2000.

[12] Z. Li, B. Li, D. Jiang, and L. C. Lau, "On achieving optimal throughput with network coding," in IEEE INFOCOM, 2005.

[13] S.-Y. R. Li, R. W. Yeung, and N. Cai, "Linear network coding," IEEE Trans. Inf. Th., vol. 49, pp. 371-381, 2003.

[14] T. Ho, M. Medard, R. Koetter, D. R. Karger, M. Effros, J. Shi, and B. Leong, "A random linear network coding approach to multicast," IEEE Trans. Inf. Th., vol. 52, no. 10, pp. 4413-4430, October 2006.

[15] D. S. Lun, N. Ratnakar, M. Médard, R. Koetter, D. R. Karger, T. Ho, E. Ahmed, and F. Zhao, "Minimum-cost multicast over coded packet networks," IEEE Trans. Inf. Th., vol. 52, no. 6, pp. 2608-2623, 2006.

[16] S. Y. Cho and C. Adjih, "Wireless broadcast with network coding in mobile ad-hoc networks: Dragoncast," http://hal.inria.fr/inria-00292867/en/, Tech. Rep., 2008.

[17] D. Silva and F. R. Kschischang, "Rank-metric codes for priority encoding transmission with network coding," in Proc. CWIT'07, june 2007.

[18] D. Silva, F. R. Kschichang, and R. Kötter, "A rank-metric approach to error control in random network coding," IEEE Trans. inf. th., vol. 54, no. 9, pp. 3951-3967, 2008.

[19] M. Shao, X. Wu, and N. Sarshar, "Rainbow network flow with network coding," in Proc. NETCOD'08, 2008, pp. 1-6.

[20] S. Dumistrescu, M. Shao, and X. Wu, "Layered multicast with interlayer network coding," in Proc. INFOCOM'09, 2009.

[21] M. Kim, D. Lucani, X. Shi, F. Zhao, and M. Médard, "Network coding for multi-resolution multicast," in Proc. INFOCOM'10, 2010.

[22] J. M. Walsh and S. Weber, "A concatenated network coding scheme for multimedia transmission," in Proc. NetCod'08, 2008.

[23] D. Vukobratovic and V. Stankovic, "Unequal error protection random linear coding for multimedia communications," in Proc. MMSP, SaintMalo, France, 2010

[24] H. Park, N. Thomos, and P. Frossard, "Transmission of correlated information sources with network coding," in EUSIPCO 2010, Aalborg, Denmark, August 2010.

[25] V. Goyal, J. Kovacevic, and J. Kelner, "Quantized frame expansions with erasures," Appl. Comput. Harmon. Anal, vol. 10, no. 3, pp. $203-$ 233, May 2001.

[26] V. K. Goyal, M. Vetterli, and N. T. Thao, "Quantized overcomplete expansions: Analysis, synthesis, and algorithms," IEEE Trans. Inf. Th., vol. 44, pp. 16-31, 1998

[27] P. A. Chou, Y. Wu, and K. Jain, "Practical network coding," in Proc. Allerton Conf. Comm., Control, and Computing, 2003.

[28] R. Fourer and D. Gay, The AMPL Book. Duxbury Press, 2002.

[29] ILOG CPLEX 11.0 User's Manual. 